Meta

Journal des tradlucteurs

Translators' Journal

\title{
Développement des langues africaines : De Yaoundé à Zanzibar
}

\section{André Clas et Jean Baudot}

Volume 29, numéro 2, juin 1984

URI : https://id.erudit.org/iderudit/002291ar

DOI : https://doi.org/10.7202/002291ar

Aller au sommaire du numéro

Éditeur(s)

Les Presses de l'Université de Montréal

\section{ISSN}

0026-0452 (imprimé)

1492-1421 (numérique)

Découvrir la revue

Citer cette note

Clas, A. \& Baudot, J. (1984). Développement des langues africaines : De Yaoundé à Zanzibar. Meta, 29(2), 212-215. https://doi.org/10.7202/002291ar d'utilisation que vous pouvez consulter en ligne.

https://apropos.erudit.org/fr/usagers/politique-dutilisation/ 
BLOC-NOTES

\section{DÉVELOPPEMENT DES LANGUES AFRICAINES : DE YAOUNDÉ À ZANZIBAR}

On a très souvent tendance à oublier, en parlant de l'Afrique francophone ou de l'Afrique anglophone, que les Africains ont leurs langues à eux et qu'ils s'en servent.

L'Afrique, et c'est elle qui le déclare, veut intégrer les langues africaines dans le développement des pays et faire en sorte qu'elles puissent assumer de plus en plus, et de mieux en mieux, leurs fonctions communicatives sur tous les plans. L'Afrique veut faire accéder, "sans détour ni sélection linguistique ", ses populations au progrès social, politique, économique et technique.

Bien entendu, on va objecter le nombre important de langues et la difficuité de la tâche, puisque la question n'est pas simplement linguistique, mais encore économique et surtout politique.

Il est vrai que le nombre de langues en Afrique est important et qu'il varie selon les pays. C'est ainsi que pour prendre quelques exemples, on peut citer le Bénin, le Burundi, le Sénégal, le Cameroun et la Tanzanie. Ainsi, au Bénin (CONFEMEN), on a recensé 52 langues nationales qui appartiennent presque toutes aux groupes gur et kwa de la famille Niger-Congo. Dix-neuf de ces langues, soit le bariba, le dendi, le fon, le yoruba, le waama, l'adja, le yom, le ditammari, le fulfudé, le boko, le gun, le tofin, l'aizo, le mokole, le lepka, le waci-gen, le nateni, l'anii et le sahwe, font actuellement l'objet d'une promotion. II faut signaler cependant que la répartition des locuteurs du fon, du gun, de l'adja, du watchi, de l'aïro et du mina s'établissait, en 1969 , à $63 \%$ de la population. Au Burundi, la situation est beaucoup plus simple puisque toutes les langues parlées sont des langues bantoues de la famille linguistique Niger-Congo et que $95 \%$ des locuteurs ont comme langue maternelle le kirundi et que beaucoup connaissent le kiswahili, langue bantoue interafricaine, apparentée. Il faut noter encore que le kinyarwanda du Rwanda est aussi apparenté au kirundi. Au Sénégal, six langues ont le statut officiel de langues nationales, soit le wolof, le poular, le sérère, le diola, le manding et le soninké. Mais il faut signaler que $80 \%$ de la population utilise le wolof. Au Cameroun, la situation est extrêmement complexe et les premières recherches dénombrent environ 130 langues différentes, mais aussì un certain nombre de langues qui ont une fonction véhiculaire, soit le fulfudé, le béti (ewondo-bulu), le duala, le hausa, etc., sans oublier un pidgin-english avec deux variétés. En Tanzanie, la langue nationale est le swahili, originaire de Zanzibar, langue bantoue utilisée par plus de 50 millions d'habitants et qui constitue, pour une grande partie de l'Afrique de l'Est, la langue véhiculaire puisqu'elle s'utilise en Tanzanie, au Kénya, en Somalie, en Uganda, au Mozambique et aux Comores, sans oublier le rôle qu'elle joue au Zaïre, au Burundi et au Rwanda.

Mosaïque linguistique? Oui, mais, même si les situations sont difficiles, elles ne sont cependant pas inextricables.

Tout d'abord, une même langue peut être utilisée dans plusieurs pays, soit comme langue maternelle soit comme langue véhiculaire, puisque les frontières linguistiques ne correspondent pas aux limites politiques. Ensuite, beaucoup de langues appartiennent à la même famille de langues, et, il ne faut pas le nier, toutes les communautés linguistiques ne sont pas d'égale force, parfois pour des raisons historiques, mais souvent aussi tout simplement à cause de facteurs démographiques. $\mathrm{Xl}$ existe donc un pouvoir d'attraction de ces langues. Ce regroupement linguistique sera d'autant plus facile que la plupart des Africains sont bilingues ou même polyglottes. C'est ainsi qu'on peut noter l'expansion du wolof au Sénégal et en Gambie, du manding avec ses dialectes le malinké, le bambara et le jula, au Mali, en Haute-Volta, en Côte d'Ivoire, en Guinée, en Sierra Leone, en Guinée Bissau et au Ghana (Calvet 1981 : 27).

Nous avons aussi dit que le nombre de locuteurs n'était pas indifférent. Sans avoir de chiffre précis, on peut estimer, par exemple, à plus de 70 millions les locuteurs du yoruba, et à des dizaines de 
millions ceux du haussa, de l'ibo, du lingala, du baulé, etc.

La question du développement des langues africaines n'est pas neuve, mais elle mérite d'être soulevée de temps à autre, surtout qu'un grand pas en avant vient d'être fait : l'organisation de cours de traduction, la création d'associations de traducteurs et d'interprètes, l'organisation de cours de terminologie (notamment à l'EACROTANAL à Zanzibar auquel les soussignés ont eu l'honneur de participer en décembre 1983), et surtout la publication d'une première série de lexiques.

En effet, dès 1967, les participants au IX Colloque international sur les langues secondes à Tunis (Houis 1971 : 177) formulaient les recommandations suivantes : "Le colloque estime que l'effort poursuivi, dans de nombreux pays du Tiers Monde, en faveur de l'apprentissage des langues secondes, devrait s'accompagner, simultanément, d'un effort comparable au profit de la langue nationale de ces pays. Sans cet effort, au processus d'enrichissement mutuel de deux cultures, justification du bilinguisme, se substituerait un processus de désaffection des maîtres et de leurs élèves vis-à-vis de leur langue et de leur culture propres, ainsi qu'une mauvaise assimilation de la culture étrangère. "

Les réunions se succèdent, on va de lavant. "La promotion des langues nationales n'est pas un enjeu théorique : elle s'inscrit dans le cadre concret du développement des peuples et de leur combat pour la reconnaissance de leur identité " peut-on lire dans l'A vant-propos du rapport de la CONFEMEN, et on sait que l'Agence de coopération culturelle et technique, qui a organisé la Conférence internationale de Bujumbura en 1976 et celle de Yaoundé en 1983 sur la promotion des langues nationales et qui a comme un de ses objectifs "l'affirmation, la sauvegarde et la promotion des identités culturelles par la revalorisation des langues nationales ", est un des maîtres d'oeuvre du développement des langues africaines par son action organisatrice et financière.

C'est ainsi que sous l'égide de l'ACCT, par le biais du CERDOTOLA, viennent d'être publiés, dans la série LEXIQUES THÉMATIQUES DE L'AFRIQUE CENTRALE (LETAC), les lexiques couvrant les Activités économiques et sociales pour l'ikinyarwanda (Équipe nationale du Rwanda), le sango (Institut national d'éducation et de formation - Centrafrique),

le ciluba (Équipe nationale zaïroise - Zaïre), le kiswahili (Équipe nationale zaïroise - Zaïre), le monokutuba (Équipe nationale zaïroise - Zaïre) Mais avant d'examiner ces lexiques, il est peut-être intéressant de rappeler les recommandations qui ressortent de la réunion des Spécialistes africains patronnée par l'Unesco, en 1982, et qui proposent :

1. De prendre toutes mesures pour mettre en oeuvre la recommandation de Nairobi, notamment dans le domaine de l'organisation de la profession de la traduction, de la formation des traducteurs et de la terminologie en Afrique ;
2. De mettre tout en oeuvre pour améliorer la traduction dans les médias, qui s'ouvrent aux masses populaires d'Afrique ;

3. De créer les cadres et structures nécessaires à la traduction des oeuvres scolaires en langues nationales;

4. De créer les cadres et structures nécessaires à la traduction scientifique et technique en langues nationales;

5. D'assurer les moyens nécessaires à la recherche terminologique nécessaire à cette activité de traduction et d'en informer INFOTERM :

6. De collecter les oeuvres de la tradition orale, condition essentielle pour la présentation d'oeuvres africaines à insérer dans la collection UNESCO des oeuvres représentatives ;

7. D’adopter un système général de transcription pour permettre la traduction de ces oeuvres et assurer ainsi leur diffusion en Afrique et dans le monde;

8. De subventionner la traduction d'oeuvres nationales importantes et de créer des prix de la traduction pour stimuler l'édition de ces oeuvres :

9. De faire un effort spécial pour l'interprétation des cultures d'Afrique et d'Asie;

10. D'envisager l'insertion dans la collection UNESCO des oeuvres représentatives des oeuvres africaines importanies;

11. De promouvoir la création d'associations de traducteurs qui devront se grouper dans des structures régionales pour intensifier leur action et s'affilier à la Fédération Internationale des traducteurs, représentant reconnu de la profession sur le plan international ;

12. Aux gouvernements d'accorder aux traducteurs la protection légale prévue à la recommandation précitée ;

13. De créer des écoles de traducteurs avec le concours des instituts existant en Afrique et ailleurs ;

14. D'assurer la formation des cadres enseignants nécessaires en recourant notamment aux professeurs de langues, aux praticiens de la traduction en Afrique et aux linguistes;

15. De favoriser des stages pour améliorer la technique de la traduction auprès des grands services de traduction nationaux ou internationaux ;

16. D'inscrire dans les accords de Coopération culturelles des projets de collaboration et de formation dans le domaine de la traduction.

17. D'inviter la Fédération internationale des traducteurs à intensifier son action en Afrique ;

18. D'inviter la Fédération internationale des traducteurs à réserver une place plus 
large dans sa revue Babel à la traduction en Afrique;

19. Aux gouvernements des États africains, à l'UNESCO et à toutes les autres organisations intéressées, d'appuyer la réalisation de ces objectifs par tous les moyens législatifs et autres dont ils disposent et notamment en mettant à la disposition des organismes appelés à les exécuter les ressources nécessaires à la réalisation de ces projets.

Si l'on examine à présent les lexiques des diverses langues traitant d'activités économiques et sociales, on est frappé par la similitude de la démarche créative suivie dans le développement du sango, de l'ikinyarwanda, du ciluba, du kiswahili, du monokutuba et dans la formation du vocabulaire de l'aviation (Guilbert 1965) ou dans la formation du vocabulaire des chemins de fer en France (Wexler 1955). On sait que dans ces deux derniers ouvrages, Guilbert et Wexler montrent "comment à un signifié nouveau vient s'attacher un signifiant nouveau "et " comment s'opère la différenciation du lexique d'une technique naissante ". On peut aussi voir que le vocabulaire technique se forme par la réunion de lexèmes provenant d'autres domaines, mais qui finissent par acquérir une nouvelle autonomie dans un nouveau cadre. Le processus de dénomination fait intervenir la paraphrase (comparaison, analogie), la spécialisation d'un terme transféré, la création morphologique, la création syntagmatique et l'emprunt. Rappelons ici que l'emprunt n'a rien de scandaleux, qu'il est même " un des mécanismes fondamentaux de l'évolution des langues ", à condition, comme on le dit si bien (LETAC, Avant-propos, p. ix), qu'« Une des tâches essentielles pour favoriser le dynamisme d'une langue consiste donc non à s'opposer à l'emprunt - ce qui irait à l'encontre d'un comportement sociolinguistique naturel - mais à en canaliser l'efflorescence par la normalisation. "C'est ainsi que les lexiques cités comportent "des mots existants et des emprunts fortement intégrés ", ainsi que des propositions concertées pour des néologismes sémantiques et (ou) lexicaux (LETAC, Introduction, p. xi). français

Citons quelques exemples d'emprunts au

1. par le sango (langue à six tons, orthographiquement marqués par l'accent circonflexe, le tréma et la répétition de la voyelle) :

kalorii $\quad$ calorie

kilöo pesée (kilo)

vitamîni vitamine

akarîome aquarium

firigöo réfrigérateur (frigo)

ti firigöo frigorifique

koperativi coopérative

mikorobo microbe

2. par l'ikinyarwanda (langue à quatre tons, orthographiquement marqués par l'accent circonflexe, le tréma et l'accent circonflexe inversé) :

$\begin{array}{ll}\text { ipŏmpa } & \text { pompe } \\ \text { segitëri } & \text { secteur } \\ \text { makète } & \text { maquette } \\ \text { maketïsiti } & \text { maquettiste } \\ \text { fagitĭre } & \text { addition, facture } \\ \text { par le ciluba (langue à tons) : } \\ \text { wikèndè } \quad \text { week-end } \\ \text { mfòlòmaàjà } & \text { fromage } \\ \text { pòlòtèyinà } & \text { protéine } \\ \text { 'emprunts à l'anglais par le kiswahili (swa- }\end{array}$

hili)

$\begin{array}{ll}\text { hoteli } & \text { hôtel } \\ \text { enzaimu } & \text { enzyme } \\ \text { ekolojia } & \text { ecology }\end{array}$

$$
\text { kolojeni collagen }
$$

Comme on peut le constater tous ces emprunts sont parfaitement intégrés, phonétiquement et orthographiquement, aux différentes langues. Mais la question de l'emprunt ne se limite pas au français et à l'anglais, mais touche en fait, comme pour n'importe quelle langue dans n'importe quel pays, aussi aux emprunts aux langues voisines et véhiculaires. Ainsi le sango dit mbârâtâ pour désigner le cheval : emprunt à l'arabe, le ciluba parle de $k a b a$ lii (portugais : cavalo) ou de mfulansa (swahili : farasi) et l'ikinyarwanda a adapté ifaräsi (swahili : farasi).

Les formations par calque ou par périphrase abondent aussi. On sait d'ailleurs que c'est un procédé de formation universel. Ainsi le sango désigne l'apiculteur par wabätängö-wôtoro, c'est-à-dire " celui élevage abeille ", traduit expérimentation par tärängö-yê, littéralement "essayage-chose ", vétérinaire par wangaga tî ânyama " guérisseur animaux", insecticide par ngûyorö "eau-médicament" et éditorialiste par wandâsango " celui-éditorial ", où éditorial est ndâsango "fond-nouvelle". Si l'on choisit quelques exemples en ikinyarwanda, on peut citer la belle création imfashisha-matsiko, littéralement " qui excite la curiosité ", donc la documentation ou encore umukurú w'igihugú, "le grand pays", c'est-àdire le président, chef de l'État.

Les procédés sont donc les mêmes que ceux que l'on peut trouver dans toutes les langues, en fonction bien entendu du système linguistique. Ainsi si en kiswahili, je crée elimu "knowledge ", je peux le joindre à uyoga "mushroom" pour obtenir elimuuyoga "mycology" ou avec umbo "shape", elimuumbo " morphology".

La créativité n'est, dans aucune langue, impossible, elle peut être plus facile dans une langue que dans une autre, mais le principal problème est bien entendu l'entente sur une stratégie commune par les divers créateurs. Les premiers pas sont faits. Il s'agit à présent d'écrire, de traduire et de diffuser, la voie est ouverte.

En conclusion, il faut rappeler qu'il n'est pour aucun pays question "d'évacuer les langues européennes ", selon la formule de Maurice Houis, mais de rechercher une complémentarité dans le respect des cultures et des uns et des autres. "Les lan- 
gues, comme les civilisations se suffisent rarement à elles-mêmes " disait Sapir.

\section{ANDRÉ Clas et JeAN BAUdot}

\section{BIBLIOGRAPHIE}

AKLILU, Amsalu (1982) : "Principles for the Creation of New Science and Technology Terms in Amharic ", in Ethiopian Studies.

AGENCE DE COOPÉRATION CULTURELLE ET TECHNIQUE (ACCT) (1983) : la Recherche linguistique, l'emploi et l'enseignement des langues en Afrique: les moyens de renforcer la coopération entre les états. Actes de la Conférence internationale, Paris, Yaoundé, ACCT et CERDOTOLA.

CALVET, Louis-Jean (1981) : les Langues véhiculaires, Paris, PUF, Que sais-je? no 1916.

CONFÉRENCE DES MINISTRES DE L'ÉDUCATION DES PAYS D'EXPRESSION FRANCAISE (CONFEMEN) (1982-1983) Promotion et intégration des langues nationales dans les systèmes éducatifs: Bilan et inventaire, Québec, ministère de l'Éducation, 2 volumes.

CONSEIL INTERNATIONAL DE LA LANGUE FRANCAISE (CILF) (1978) : Inventaire des études linguistiques sur les pays d'Afrique Noire d'expression française, Paris, CILF.

GIBBE, A.G. (1981) : "The Development of Kiswahili Technical Terms", Studies \& Documents, vol. 2, EACROTANAL, pp. 17-33.

GREENBERG, Joseph H. (1970) : The Languages of Africa, Bloomington, The Hague, Indiana University Press, Mouton, $1^{\text {st }}$ ed. 1966, $3^{\text {rd }}$ ed.

GUILBERT, Louis (1965) : la Formation $d u$ vocabulaire de l'aviation, Paris, Larousse.

HOUIS, Maurice (1971) : Anthropologie linguistique de l'Afrique Noire, Paris, PUF, La linguistique no 11.

RAHAINGOSON, Henri (1981) : la Mise en place d'une langue d'enseignement : l'exemple malgache (polycopié), EACROTANAL, Zanzibar.

TEMU, C.W. (1982) : Kiswahili Terminology (Principles adopted for the enrichement of the kiswahili language) (preprint), EACROTANAL, Zanzibar.

WALUSIMBI, L. (1983) : Linguistic Terminology in Luganda (preprint) EACROTANAL, Zanzibar.

WEXLER, P.J. (1955) : la Formation du vocabulaire des chemins de fer en France (1778-1842), Genève, Droz.

WHITELEY, Wilfred (1969) : Swahili, The Rise of a National Language, London, Methuen. 
Québec Science) a fait état de l'envahissement du monde scientifique par les publications américaines et de la difficulté que l'on éprouve à se procurer la terminologie française dans les domaines de pointe, les lancements de la NASA par exemple. Les scientifiques sont de plus en plus conscients des problèmes terminologiques et font un effort pour utiliser des termes justes. Québec-Science essaie en outre de doser la quantité de nouveaux termes et de les définir au fur et à mesure dans les articles afin de suivre la politique de vulgarisation de la maison.

Dans le secteur de la publicité, Gilles Tremblay (Langagerie inc.) a exposé les modes de formation des néologismes et a montré comment les termes publicitaires ou créés par la publicité influent sur la langue courante.

En ce qui concerne la diffusion de la terminologie par les documents de l'entreprise, Pierre Gagnon (Westinghouse) a parlé de la fierté que ressentaient les employés à travailler avec des outils en français ainsi que de la difficulté à travailler avec des documents normatifs plus ou moins bien traduits.

La francisation de l'informatique pose certains problèmes qui ne sont pas insolubles cependant, comme l'a expliqué Patricia Labrie (planification, section française, IBM). Il peut être assez aisé de traduire des manuels d'utilisation d'appareils, mais lorsqu'il faut traduire des progiciels ou des informations écran, la francisation prend une tout autre dimension. En effet, avant de concevoir un produit, les ingénieurs doivent vérifier si celui-ci est appelé à être traduit et le structurer de façon qu'il soit exploitable en français. Ensuite, la traduction proprement dite est effectuée, suivie de la validation du produit français en laboratoire. Les services de traduction d'IBM France et d'IBM Canada se partagent les traductions, uniformisent la terminologie selon le pays qui est le plus avancé dans le domaine, et adaptent selon la réglementation en vigueur. IBM pense d'ailleurs commercialiser un outil de traduction pour l'information écran.

La deuxième journée a été consacrée à l'examen des moyens et des solutions, puis à la prospective.

Les textes normatifs de nature juridique ne sont-ils pas des moyens de diffusion de la terminologie? Antoni Dandonneau (Commission des valeurs mobilières du Québec) a montré que la loi et le règlement contribuent à accréditer la légitimité des termes qu'ils emploient, même si ces termes, dont le monde du droit - et parfois le public - est " imprégné ", sont souvent fautifs. Pour déloger ces termes, il faut changer la loi. La nouvelle Loi sur les valeurs mobilières en est un excellent exemple, et la terminologie correcte qu'elle contient se répand de plus en plus, tandis que l'ancien vocabulaire tombe dans l'oubli. Cependant, l'État devrait manifester plus vigoureusement sa volonté de francisation, en imposant dans tous les textes de loi une terminologie de qualité.

Pour améliorer la qualité du français parlé et écrit de ses professeurs et de ses élèves, l'École québécoise du meuble et du bois ouvré (Cégep de Victoriaville) a réalisé "l'intégration des langues de spécialité dans la formation professionnelle "qu'elle donne en production de meubles, rembourrage, fabrication de gabarits et de prototypes, dessin technique et conception de mobilier. Richard Côté, professeur, et Reynald Binette, terminologue, ont expliqué que, dans ce contexte, le défi à relever consistait à fournir aux enseignants et aux élèves une contrepartie française aux manuels de formation anglo-américains qui soit fidèle aux normes et à la technologie nord-américaine. Un tel projet posait deux grands problèmes : l'uniformisation de la terminologie et la technique de rédaction. Avec l'appui de l'OLF, M. Binette a rédigé un "Vocabulaire du rembourrage", quj a servi à $\mathbf{M}$. Côté pour la rédaction de son manuel. Quatre autres vacabulaires ont suivi, et un sixième est en cours, ainsi qu'un autre manuel. L'École du meuble est maintenant le foyer d'une grande activité terminologique qui rayonne dans toute l'industrie et dont le succès à long terme dépend de l'engagement des professeurs et des élèves, futurs membres des divers corps professionnels.

Madame Hélène Cajolet-Laganière a ensuite traité du "rôle des producteurs de documentation technique dans la diffusion de la terminologie ". La documentation technique et scientifique peut être produite par l'entreprise, par ses clients et fournisseurs, ou il peut s'agir de documentation de référence (normes, ouvrages techniques, etc.). Les rédacteurs techniques, qui occupent généralement le rôle de cadres intermédiaires dans l'entreprise, doivent trouver la terminologie existante, l'adapter, la compiler, la diffuser et l'implanter. Ils rencontrent cependant plusieurs obstacles : méconnaissance de la terminologie, contraintes de temps et d'argent, et surtout manque d'intérêt. La "volonté politique "et la " fierté du travail bien fait " sont en effet indispensables à l'utilisation par les producteurs de documents techniques d'une terminologie française uniformisée, tout spécialement dans les secteurs-clés de l'économie de demain.

Quel est donc le rôle des publications terminologiques (lexiques, vocabulaires, fiches, etc.)? Charles Dupont (Air Canada) a tout d'abord traité de leurs rôles sociaux généraux : prouver l'existence de la terminologie dans les domaines techniques et scientifiques, affirmer l'identité culturelle et linguistique d'un groupe, appuyer les interventions de l'État en matière de langue. Leurs rôles sociaux spécifiques consistent à : permettre la communication entre grands usagers des langues de spécialité, faciliter la vulgarisation de la terminologie, être un instrument pédagogique, être un moyen de pression, et appuyer l'édition. Les rôles linguistiques généraux de ces publications sont les suivants : répertorier des terminologies propres à des domaines techniques et scientifiques; préserver le caractère idjomatique des langues de spécialité ; participer au développement de la langue des sciences et des techniques au même rythme que leur évolution. Quant à leurs rôles linguistiques spécifiques, ils sont, toujours d'après $M$. Dupont, d'analyser la terminologie propre à un groupe organisationnel, de modifier des habitudes langagières, de contribuer à l'uniformisation de la terminologie et d'orienter l'usage. 